\title{
A multiplexed CW Brillouin system for precise interrogation of a sensor array made from short discrete sections of optical fibre
}

\author{
J. P. Dakin ${ }^{\mathrm{a}}$, S. Chin ${ }^{\mathrm{b}}$ and L. Thévenaz ${ }^{\mathrm{b}}$ \\ ${ }^{a}$ Emeritus Professor, Optoelectronics Research Centre, University of Southampton, \\ ${ }^{b}$ Ecole Polytechnique Fédérale de Lausanne, STI-NAM Station 11, CH-1015 Lausanne, Switzerland \\ ipd@orc.soton.ac.uk, sanghoon.chin@epfl.ch, luc.thevenaz@epfl.ch
}

\begin{abstract}
A novel configuration for precise temperature sensing in an array of optical fibre sections is reported. The sections are chosen to have different Brillouin shifts, to enable separation of scattered light signals in the frequency domain, rather than the traditional time domain, allowing lower-noise CW operation and greater precision due to the $100 \%$ duty cycle. Keywords: Instrumentation, Fiber-optic sensor, Optical fibers, Brillouin scattering, temperature sensing.
\end{abstract}

\section{INTRODUCTION}

Raman and Brillouin scattering are both commonly-used, truly-distributed methods of interrogating long lengths of fibre [1-4]. Brillouin scattering is narrow band in nature, and the frequency difference between incident and scattered light is a function of temperature, strain and fibre properties, particularly the core material $[5,6]$. Whilst a powerful tool for sensing, the instrumentation for distributed Brillouin sensing is complex, and the precision is limited by the need to use pulsed light signals. In this work, we take advantage of the fact that the Brillouin shift varies with fibre properties, allowing construction of a sensing array by splicing several different fibre together (each with significantly different Brillouin shifts), addressing the array with a continuous wave (CW) optical signal, and separating signals in the frequency domain.

\section{PRINCIPLE AND APPARATUS}

Brillouin scattering is usually described as the light scattered by thermally excited acoustic phonons and the Brillouin shift $v_{\mathrm{B}}=2 n V_{\mathrm{a}} / \lambda$ depends on the velocity, $V_{\mathrm{a}}$, of the longitudinal acoustic wave, the effective refractive index, $\mathrm{n}$, of the fibre and the wavelength of light $\lambda$ [6]. The first two parameters are dependent on fibre composition. Our new sensing arrangement is shown in Fig.1. When the fibre sensing array is excited with a CW optical incident signal, a set of different scattered signals is generated, of optical frequency depending on the fibre type and material. For a given fibre section, however, the exact frequency of each scattered signal also reflects the physical condition of this section, particularly the temperature of, and mechanical strain in, the fibre. If the fibre sections are packaged in loosely-wound coils, to avoid strain, then a useful temperature sensing array is formed. It is only a multiplexed system, rather than being fully distributed, but the sensor is now capable of better s/n ratio, allowing far greater real-time measurement precision.

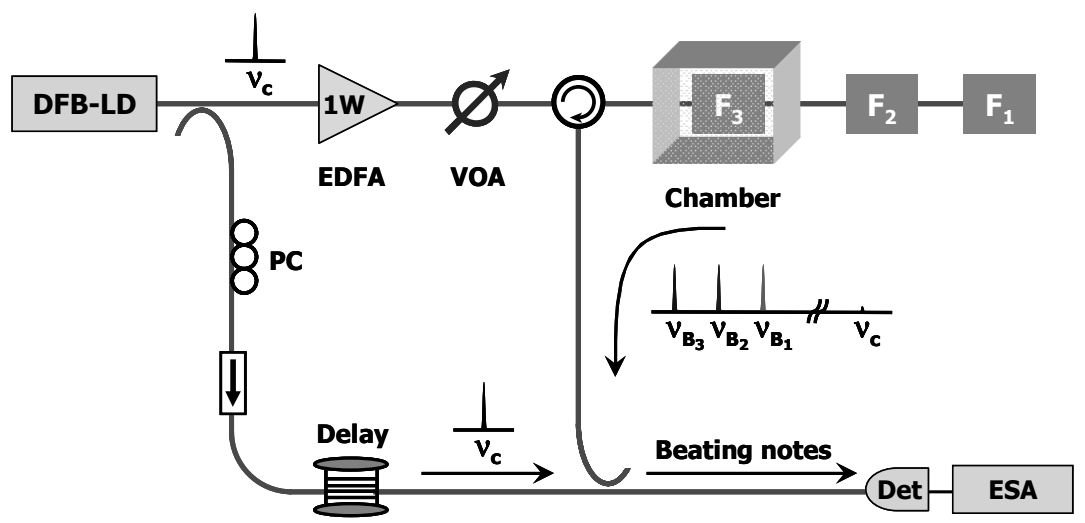

Fig.1: Schematic of apparatus for first viability tests. EDFA is Erbium Doped Fiber Amplifier; VOA is Variable Optical Attenuator; Det is Detector and ESA is Electrical Spectrum Analyzer

19th International Conference on Optical Fibre Sensors, edited by David Sampson, Stephen Collins, Kyunghwan Oh, Ryozo Yamauchi, Proc. of SPIE Vol. 7004, 70046L, (2008) 0277-786X/08/\$18 doi: 10.1117/12.801233 

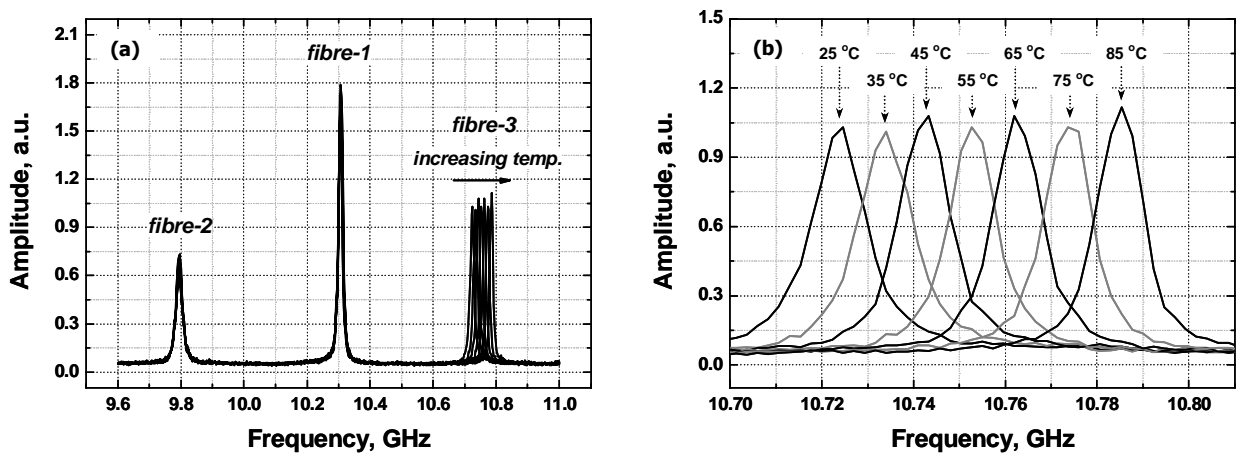

Fig.2: RF spectra of Brillouin signals from the 3 fibre sections (with expanded scales on the right hand side)

In our system, the optical signals are arranged to return to a single optical detector, along with a local oscillator signal, which is derived by tapping off part of the signal used to excite the fibre sensing array. The heterodyne output of this detector is a set of beat frequencies, each depending on the Brillouin shift of the corresponding fibre sensing section and on the frequency of the local oscillator. We shall show that not only does this form a relatively simple multiplexed sensor system, but it is also one which is capable of real-time thermal sensing with high precision.

Initially, to validate the basic principle, 3 loosely-coiled optical fibres of different type (fibre-1: $50 \mathrm{~m}$ of bendinsensitive fiber; fibre-2: $15 \mathrm{~m}$ of dispersion-compensated fiber; and fibre-3: $50 \mathrm{~m}$ of dispersion-shifted fiber) were spliced to form a simple sensor array (Fig.1). The incident light source was a CW DFB semiconductor laser diode (NEL type NLK1556STG, of wavelength $1551 \mathrm{~nm}$, linewidth $\sim 1 \mathrm{MHz}$ ), amplified with a erbium-doped fibre amplifier, with a saturated output power of $30 \mathrm{dBm}$, giving a launch power of $\sim 1 \mathrm{~W}$ into the circulator. Light exits from the $2^{\text {nd }}$ port of the circulator into the sensing array, and the return backscatter signal exits from the third port. This is mixed with some of the light from the $\mathrm{CW}$ source laser on a high-speed $(>10 \mathrm{GHz}$ ) analogue optical receiver (New Focus InGaAs, $\mathrm{PIN} /$ transimpedance receiver, type NFO-1544-A with $\mathrm{NEP}=33 \mathrm{pW} / \mathrm{Hz}^{-1 / 2}$ ). The resulting electrical beat frequencies from the detector were then viewed using a conventional electrical spectrum analyzer (type HP 8563E).

The display of the spectrum analyzer (Fig.2-a) shows several RF frequency spectra, taken as the temperature of the $3^{\text {rd }}$ fibre (Brillouin shift $\sim 10.9 \mathrm{GHz}$ at $25^{\circ} \mathrm{C}$ ) is changed from $25^{\circ} \mathrm{C}$ to $85^{\circ} \mathrm{C}$, whilst the other 2 fibres were held constant. The Brillouin shift change of fibre 3, derived using the spectrum analyzer, is shown in Fig.3. The scale factor for the thermally-modulated fibre is $\sim 1 \mathrm{MHz}$ per ${ }^{0} \mathrm{C}$, whereas the signals from others stay, as expected, at constant frequency.

The amplitude of the Brillouin scattering peaks from each fibre varies, as a result of the different gain and pump power in each. Ideally the sensing segments should all have a similar gain, being just under the Brillouin threshold to

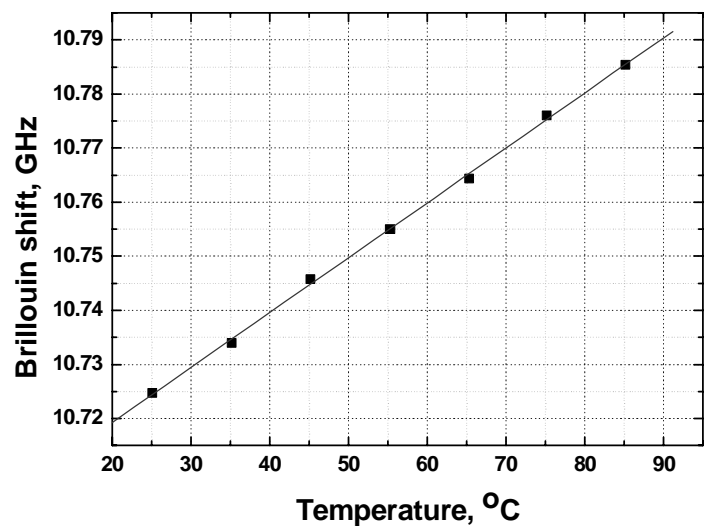

Fig.3: Plot of Brillouin shift versus temperature of fibre section 3, taken from ESA data. 
avoid signal depletion or dynamic oscillations in the system. A fluctuating pump power in a fibre segment will reduce accuracy, so we chose fibre lengths, so as to operate just below the Brillouin threshold with the pump power from our saturated amplifier. As the fibres had different materials and core sizes, the length of each segment was different.

Please note that, in Fig.1, fibre which takes light directly from the laser has a delay coil of $\sim 1 \mathrm{~km}$ length, much longer than the laser coherence length, in order to suppress any noise due to (Mach-Zehnder type) interference with any stray un-shifted light from the pump. There will also be a small residual level of un-shifted (elastically scattered) stray light because the components do not have ideal behaviour, and therefore such light can return from the $3^{\text {rd }}$ port of the circulator and interfere, on the detector, with the by-passed pump light used as our local oscillator signal.

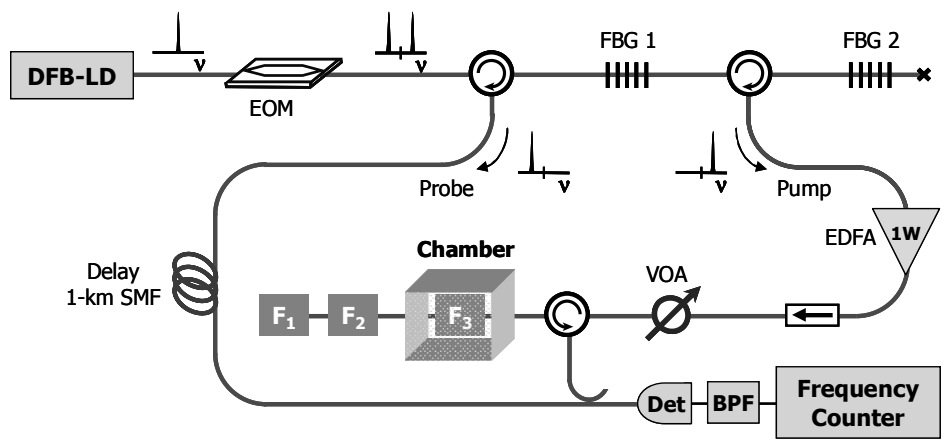

Fig.4: Schematic diagram of the real-time frequency measurement system $\left(2^{\text {nd }}\right.$ electrical mixing down stage not shown).

Having demonstrated the viability of the method, we then constructed a $2^{\text {nd }}$ system (Fig.4), where we mixed down the Brillouin-shift heterodyne signals with a local oscillator (This L.O is a modulation sideband of the pump source after filtering out the carrier and other sidebands with fibre gratings) and fed the baseband signal to a frequency counter, to enable more precise readout of mean frequency, and hence temperature. If the signal were a perfect sinusoid, then the precision, as a fraction of the initial $10 \mathrm{GHz}$ signal, would be $\sim 10^{-10}$ with only $1 \mathrm{~s}$ measurement time! Unfortunately, in practice, such high precision is not achievable, as errors may occur due to: (a) random variations in mean frequency, as a result of the finite Brillouin linewidth (b) frequency variations of the laser source and of the oscillator used to mix-down the Brillouin signals, and (c) intensity noise, from laser-source RIN, receiver noise or photon noise, all of which, if severe, can combine to cause occasional missed or even spurious additional cycle counts. We believe the errors for the DFB laser frequency fluctuations are, however, likely to be small, as $\sim 1.4 \mathrm{~nm}$ wavelength shift required to cause $10 \mathrm{kHz}$ error.

In the system of Fig.4, it was convenient to do the $1^{\text {st }}$ heterodyning stage (mixing down from the high Brillouinshift frequencies) at optical frequencies, as this allows use of a low-frequency optical receiver, having lower cost and superior spectral noise density to a $10 \mathrm{GHz}$ one. Mixing directly in the detector to baseband frequencies, was found to

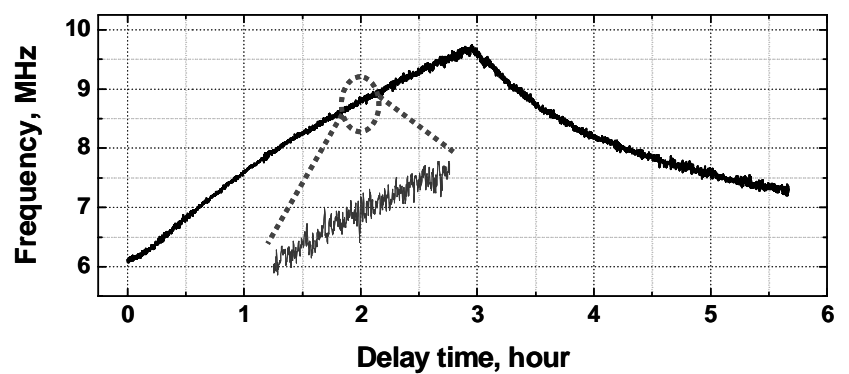

Fig.5: Frequency of mixed-down signals as measured on counter, during slow heating and cooling cycle, with temperature excursion of $\sim 3.5 \mathrm{~K}$. 
give significant low frequency noise, so we mixed in two stages, first optically to an intermediate detected RF frequency $\sim 145 \mathrm{MHz}$, then electrically to a 0 --> $15 \mathrm{MHz}$ baseband (low-pass filter), before being passed to the frequency counter. For clarity, this $2^{\text {nd }}$ electrical down-conversion stage is not shown in Fig.4. (Please note that an alternative arrangement to this counting system would be to sample the signal and use a digital processor, to perform the well-known method of fast Fourier transform analysis, and/or other appropriate signal processing algorithms [7] to find the mean frequency). If we wish to detect the temperature of the other fibres, this can be done by extending the narrow-band post-detection system to include local oscillators, mixers etc to detect other Brillouin bands, as done in a conventional radio receiver.

As we lacked a precision oven to control the temperature of the fibres to within $0.001 \mathrm{~K}$, we determined the noiselimited precision of the method by placing the fibre in a container of high thermal capacity, surrounded by thermal insulation, to allow only very gradual thermal variation. We then slowly swept the fibre temperature over a very small interval of order $3.5 \mathrm{~K}$, by first applying thermal energy via a resistive heater and then allowing it to cool, and we logged the counter frequency as a function of time, as shown in Fig.5. We found typical short-term fluctuations of order \pm 10 $\mathrm{kHz}$ RMS, equivalent to a temperature variation of approx. $\pm 10 \mathrm{mK}$, from the scale factor in our earlier measurement, an excellent value considering the short ( 1 second) acquisition time for each reading. However, at times, some additional slightly-larger fluctuations appear. We are still investigating the cause of these.

The maximum number of fiber segments in the array depends on the desired temperature range, as spectral overlapping must be avoided. For a $100 \mathrm{~K}$ change, the Brillouin frequency will shift about $100 \mathrm{MHz}$, and it is possible to fabricate fibre with Brillouin centre frequencies with $\sim 1.5 \mathrm{GHz}$ frequency total spread. We believe therefore that $\sim 10$ different fiber segments are feasible over the $1.5 \mathrm{GHz}$ frequency range, once guard bands have been included to allow for the finite Brillouin linewidth. Clearly more fibre segments are possible, if a smaller temperature range is required.

\section{CONCLUSION}

We have demonstrated the initial feasibility of addressing an array of fibre sensors with a novel CW Brillouin method. Initial results suggest that real-time temperature measurements, with a noise-limited precision of order $\pm 10 \mathrm{mK}$ RMS, in a very short update period of 1 second, is possible.

\section{ACKNOWLEDGEMENTS}

We wish to thank A Sansom, of Colledge Electronics, for his kind donation of sample filters at short notice. Prof John Dakin wishes to thank EPFL for granting a short visiting professorship.

\section{REFERENCES}

[1] Dakin J P, “Distributed optical fibre sensors” SPIE critical review CR44, 162-199 (1992).

[2] Dakin J P, Pratt D J, Bibby G W and Ross J N, "Distributed optical fibre Raman Temperature Sensor using a semiconductor light source and detector," Electron. Lett. 21, 569-570 (1985).

[3] T Kurashima, T Horiguchi, H Izumita, S Furukawa, Y Koyamda, "Brillouin optical-fibre time-domain reflectometry," IEICE Trans Commun E76-B, \#4, 382-389 (1993).

[4] Horiguchi T, Kurashima T and Koyamada Y, "Measurement of temperature and strain distribution by Brillouin frequency shift in silica optical fibers," Proc. SPIE 1797, 2-13 (1992).

[5] N. Yoshizawa and T. Imai, "Stimulated Brillouin scattering suppression by means of applying strain distribution to fiber with cabling," J. Lightwave Technol. 11, 1519-1522 (1993).

[6] M. Nikles, L. Thevenaz and P. A. Robert, "Brillouin gain spectrum characterization in single mode optical fibers," J. Lightwave Technol. 15, 1842-1851 (1997).

[7] J. K. Wu, J. Long, J. X Wang and F. He, "A novel method for fundamental frequency measurement of multiharmonic signals with noises using numerical differentiation," Mechanical Systems and Signal Processing, 19, 806816 (2005). 